\title{
L'assistenza integrata della depressione in medicina generale: una rassegna delle revisioni sistematiche sulla sua efficacia
}

\author{
Collaborative care for depression in general practice: overview \\ of systematic reviews
}

Received 18 February 2010; Revised 17 May 2010; Accepted 27 June 2010

Key words: depression, general practice, systematic reviews, integrated care.

Dear Editor

La depressione è una delle principali cause di disabilità nel mondo (Lopez et al., 2006). Colpisce dal 5 al $10 \%$ degli individui ed è la terza ragione più comune di consultazione del medico di medicina generale (Singleton et al., 2001). L'impatto della depressione sulla salute generale e il funzionamento sociale e occupazionale è sostanziale. Alla depressione sono inoltre attribuibili i due terzi di tutti i suicidi (Sartorius, 2001). E' stato stimato che circa il 90-95\% dei pazienti con disturbi di tipo depressivo sono trattati solo nel setting della medicina generale (NICE, 2004). Numerosi studi epidemiologici condotti nel setting della medicina generale hanno evidenziato che circa la metà dei pazienti che manifestano sintomi depressivi clinicamente rilevanti non ricevono una diagnosi appropriata e solo ad una minoranza di essi viene proposto un trattamento adeguato (Barbui \& Tansella, 2006). Spesso i farmaci non sono prescritti in dose terapeutica e i pazienti non assumono il trattamento nelle modalità più efficaci. Il monitoraggio dei pazienti per verificare la risposta al trattamento, l'adeguatezza della assunzione e la frequenza di recidive spesso non viene effettuato in modo sistematico ed organizzato (NHS Centre for Reviews and Dissemination, 2002).

Le strategie per migliorare la gestione e il trattamento nel setting della medicina generale possono essere raggruppate in quattro categorie: formazione per i medici di medicina generale, consultazione con specialisti della salute mentale, assistenza integrata, invio a strutture di secondo livello.

L'assistenza integrata ("collaborative care" nella letteratura anglosassone) è un tipico "intervento complesso" che comprende al suo interno numerose componenti diverse che interagiscono nel determinare l'efficacia dell'intervento nel suo insieme, sebbene l'impatto di ognuna di esse possa essere difficile da individuare. Le componenti del modello della assistenza integrata, variamente combinate nelle diverse strategie di implementazione, comprendono la formazione dei medici di medicina generale, la formazione del paziente, il coordinamento e la collaborazione di diverse figure professionali nel processo di assistenza, il monitoraggio del paziente nel tempo per verificare l'adesione e la risposta al trattamento, -spesso effettuato da una figura professionale specifica, il "case manager". Tale figura è responsabile del follow up del paziente, della verifica sui livelli di adesione e della risposta alla terapia psicologica e farmacologica, della segnalazione di eventuali peggioramenti o ricadute in occasione delle quali può prendere iniziative, dell'offerta di supporto psicologico al paziente. Il case manager lavora in stretta collaborazione con il medico di medicina generale (MMG) e con uno specialista della salute mentale.

Numerose revisioni sistematiche sono state pubblicate negli ultimi anni con l'obiettivo di valutare l'efficacia della assistenza integrata. L'obiettivo di questo studio è di effettuare una rassegna sistematica delle revisioni sistematiche pubblicate sull'efficacia degli interventi integrati sintetizzandone i risultati.

Metodi: Criteri di inclusione per una chiara definizione dei criteri di inclusione e di una strategia di ricerca bibliografica accurata ci si è avvalsi del modello PICOS (Pazienti, Intervento, Confronto, Outcome, disegno di Studio) (O'Connor et al, 2008):

P: pazienti con diagnosi primaria di depressione effettuata attraverso i Research Diagnostic Criteria (RDC), il Diagnostic and Statistical Manual (DSM), i criteri della International Classification Disorder (ICD), o che sono stati valutati per la presenza di 
sintomi significativi attraverso strumenti validati, sia di self report sia somministrati dal medico. (es. Beck Depression Inventory (BDI)

I: intervento integrato (collaborative care) che prevede l'intervento del medico di medicina generale con il supporto di un approccio multiprofessionale coordinato composto da specialisti della salute mentale, infermieri specializzati, la formazione ed il supporto del paziente, il monitoraggio dei sintomi e dell'adesione al trattamento attraverso un follow up strutturato.

C: assistenza abituale effettuata dal solo medico di medicina generale: qualunque tipo di assistenza effettuata dal medico di base, che può anche prevedere l'invio a strutture specialistiche, la consulenza di specialisti o l'ausilio di un infermiere, ma non inserita in un programma strutturato e organizzato di assistenza multidisciplinare

O: riduzione dei sintomi, remissione, adesione al trattamento, appropriatezza del trattamento;

S: revisioni sistematiche della letteratura (revisioni con definizione chiara degli obiettivi, dei criteri di inclusione degli studi, della strategia di ricerca bibliografica, del numero degli studi inclusi)

Strategia di ricerca: è stata effettuata una ricerca bibliografica sulle seguenti banche dati: Medline (PubMed), banca dati biomedica prodotta dalla National Library of Medicine di Bethesda, USA, Embase, banca dati europea per le informazioni farmacologiche e tossicologiche di proprietà di Elsevier, Amsterdam, The Cochrane Library, banca dati ufficiale della Cochrane Collaboration che raccoglie le revisioni sistematiche dei gruppi Cochrane e le revisioni sistematiche pubblicate sulle principali riviste scientifiche. Sono state incluse le revisioni pubblicate in italiano, inglese, spagnolo e francese. Sono stati consultati gli anni dal 2000 al 2008 (Table 1).

Selezione degli studi: una prima selezione è stata effettuata da due revisori in modo indipendente sulla base dei titoli e degli abstracts. Le revisioni potenzialmente eleggibili sono state acquisite in full text e valutate per verificare la rispondenza ai criteri di inclusione da due revisori in modo indipendente. Eventuali disaccordi sono stati risolti tramite discussione.

Valutazione della qualità metodologica La qualità metodologica delle revisioni è stata valutata da due revisori, in modo indipendente, utilizzando la checklist Amstar (Shea et al., 2007). Eventuali disaccordi sono stati risolti tramite discussione.

Estrazione dati: Per ogni revisione inclusa è stata preparata una tabella in cui sono state riportate le informazioni relative a: obiettivo, numero e disegno degli studi inclusi, caratteristiche dei pazienti, dell'intervento sperimentale e di controllo, misure di risultato, risultati e conclusioni.

\section{Risultati}

Attraverso la ricerca bibliografica sono state individuate 47 referenze. In base ad una prima selezione, sui titoli e sugli abstracts, sono state selezionate e acquisite in full text 16 revisioni per una valutazione più approfondita. In seguito ad una analisi dettagliata dei full text sono state incluse 10 revisioni.

Revisioni escluse. Sei revisioni sono state escluse. La ragione della esclusione è stata: revisioni narrative: 2 articoli (Adli et al., 2006, Barbui \& Tansella, 2006); protocollo di revisione: 1 articolo (Fletcher et al., 2007); editoriale: 1 articolo (Katon \& Unutzer, 2006); obiettivo della revisione non pertinente: 2 articoli (Gilbody et al., 2003; Craven \& Bland, 2006)

\section{Revisioni incluse}

\section{Caratteristiche}

Sono state incluse 10 revisioni (Badamgarav et al., 2003; Vergouwen et al., 2003; Neumeyer-Gromen et al., 2004; Bower et al., 2006; Gilbody et al., 2006; Gunn et al., 2006; Gensichen et al., 2006; Williams et al., 2007; Christensen et al., 2008; Chang-Quan et al., 2009) per un totale di 75 studi primari inclusi (Tab. 2). Il numero di studi inclusi per revisione va da un minimo di 3 ad un massimo di 55. La sovrapposizione degli studi inclusi tra le varie revisioni è molto alta. La maggior parte degli studi inclusi sono stati condotti negli Stati Uniti.

I pazienti inclusi sono adulti con depressione trattati nel contesto della medicina generale. Una revisione (Chan-Quan et al., 2009) include solo studi con pazienti anziani (età $\geq 60$ anni).

In tutte le revisioni l'assistenza collaborativa viene confrontata con l'assistenza abituale; in una revisione (Chan-Quan et al., 2009) uno dei 3 studi inclusi mette a confronto 2 diversi tipi di assistenza collaborativa che differiscono principalmente per l'intensità dei controlli al paziente. Una revisione (Christensen et al., 2008) ha come obiettivo quello di valutare quali delle componenti che fanno parte degli interventi integrati siano più efficaci; le componenti considerate sono: interventi rivolti al medico (programmi formativi, programmi di implementazione di linee guida, audit and feedback, utilizzo di reminders); interventi rivolti al paziente (interventi formativi sulla natura della depressione, training per l'auto aiuto, preferenze del paziente incorporate nel programma di cura); interventi di tipo organizzativo (interventi effettuati da team interdisciplinari, gestione coordinata del processo di assistenza, follow up regolare e coordinato, monitoraggio delle condizioni del paziente e del suo grado di adesione al trattamento). 
Tabella 1. strategia di ricerca

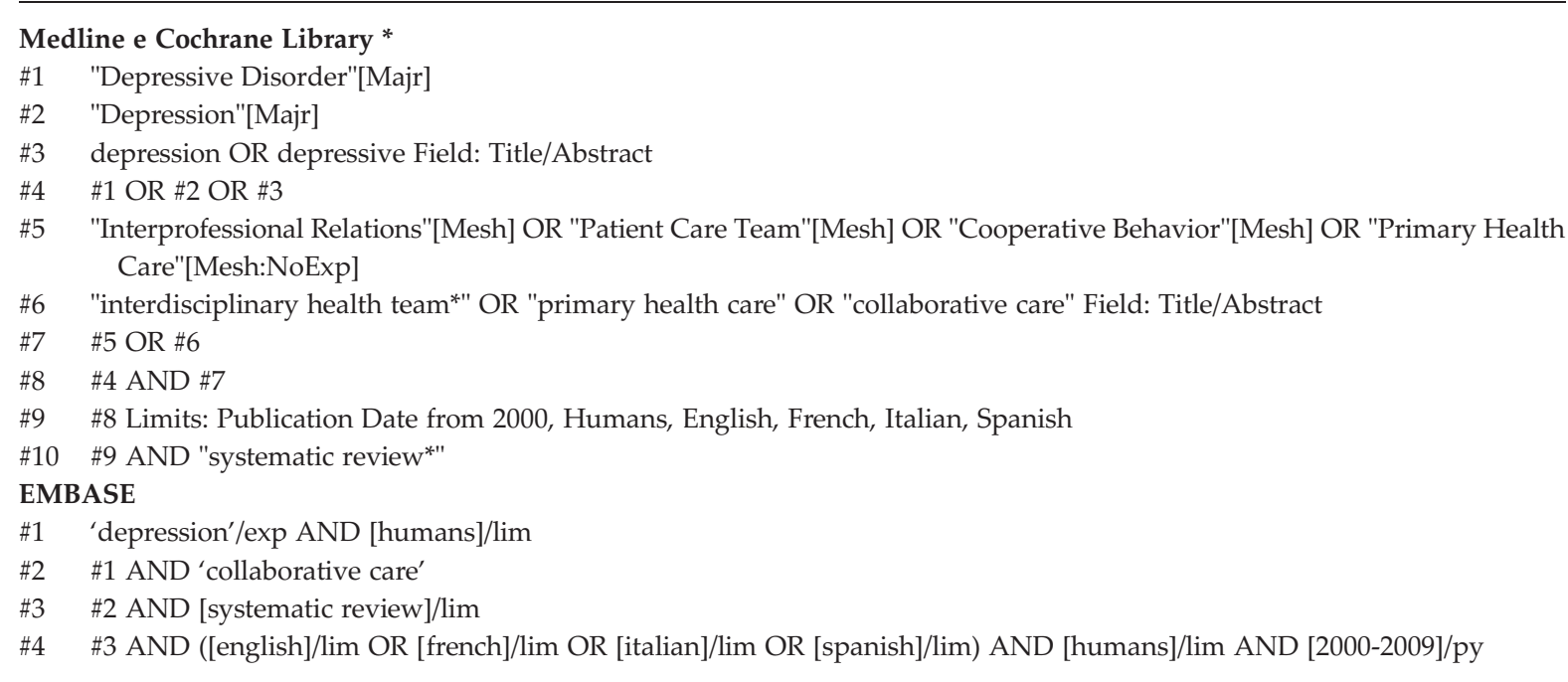

* Nella ricerca sulla Cochrane Library è stato posto solo il limite di data di pubblicazione

L'assistenza collaborativa è generalmente definita come "intervento complesso basato su un approccio multiprofessionale che vede coinvolti il MMG ed almeno un'altra figura (ie. specialista della salute mentale e/o infermiere che provvede al coordinamento e alla comunicazione tra gli operatori); può prevedere interventi di formazione e supporto del paziente, monitoraggio dei sintomi e del grado di adesione al trattamento, eventuale trattamento psicologico, utilizzo da parte dei MMG di linee guida, forme di collaborazione tra le diverse professionalità coinvolte".

Le misure di risultato considerate sono: miglioramento dei sintomi della depressione, frequenza di remissione, adesione al trattamento, gravità dei sintomi.

Sei revisioni forniscono una sintesi quantitativa dei risultati per ciò che riguarda le misure di risultato principali; in 4 revisioni (Vergouwen et al., 2003; Gunn et al., 2006; Williams et al., 2007; Christensen et al., 2008) viene fornita solo una sintesi qualitativa degli studi inclusi.

\section{Qualità metodologica}

La qualità metodologica delle revisioni incluse è di livello intermedio (Tab 3): tutte effettuano ricerche bibliografiche su più banche dati, ma solo una (Badamgarav et al., 2003) fornisce dettagli sull'esaustività delle ricerche (ricerca di letteratura grigia e studi non pubblicati, limitazioni di lingua). Sette revisioni (Neumeyer-Gromen et al., 2004; Bower et al., 2006; Gunn et al., 2006; Gensichen et al., 2006; Williams et al., 2007; Christensen et al., 2008; Chang-Quan et al., 2009) effettuano la valutazione della qualità degli studi inclusi, ma nel 30\% di esse non è chiaro come questa venga poi utilizzata nella contestualizzazione dei risultati. Solo 3 revisioni riportano la lista degli studi esclusi con le ragioni dell'esclusione (Vergouwen et al., 2003; Neumeyer-Gromen et al., 2004; Williams et al., 2007). La presenza di bias di pubblicazione è stata valutata nel $60 \%$ delle revisioni incluse.

\section{Effetti degli interventi}

Adesione al trattamento / uso di antidepressivi : cinque revisioni considerano questa misura di risultato; tre effettuano meta-analisi (Neumeyer-Gromen et al., 2004; Gensichen et al., 2006; Bower et al., 2006) e riportano risultati statisticamente significativi a favore della assistenza integrata (adesione al trattamento: RR 0.59, CI95\% $0.46-0.75$ no vs sì; RR 1.5 , CI95\% $1.28-1.86$ si vs no; uso di antidepressivi: OR 1.92, CI95\% 1.54-2.39). Delle due revisioni che non effettuano meta-analisi Vergouwen et al. (2003) riporta che $9 \mathrm{su}$ 12 studi hanno risultati in favore della assistenza integrata, Williams et al. (2007) riporta un aumento mediano di antidepressivi prescritti del $17.8 \%$ (range $9.6 \%$ $50.8 \%$ ).

Miglioramento / remissione dei sintomi : Due revisioni valutano la frequenza di remissione e riportano risultati statisticamente significati a favore dell'assistenza integrata : RR 1.39, CI95\% 1.30-1.48 (Gensichen et al., 2006); OR 2.57, CI95\% 1.61-3.33 (Chang-Quan et al., 2009). Sei revisioni effettuano meta-analisi sul miglioramento dei sintomi; Neumeyer-Gromen et al. (2004) misura il rischio di non miglioramento (RR: 0.75, CI95\% 0.70-0.81). Chang-Quan et al. (2009) misura il 
Tabella 2. caratteristiche delle revisioni incluse e risultati

\begin{tabular}{|c|c|c|c|c|}
\hline Revisione & Studi inclusi & $\mathrm{N}^{\circ}$ pazienti & Interventi & Risultati \\
\hline Badamgarav et al. 2003 & 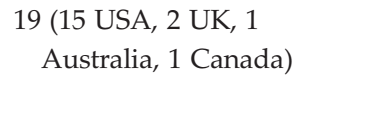 & 13220 & $\begin{array}{l}\text { Assistenza collaborativa (disease } \\
\text { management programs - DMPs) vs } \\
\text { Assistenza abituale }\end{array}$ & $\begin{array}{l}\text { Miglioramento Sintomi della depressione SMD } 0.33 \text { (IC 95\% 0.16-0.49) } \\
\text { Adesione al trattamento: SMD: } 0.36 \text { (IC 95\% 0.17-0.54) }\end{array}$ \\
\hline Vergouwen et al. 2003 & $\begin{array}{l}11 \text { paesi di realizzazione } \\
\text { degli studi non riportati }\end{array}$ & 5232 & $\begin{array}{l}\text { Assistenza collaborativa vs Assistenza } \\
\text { abituale }\end{array}$ & $\begin{array}{l}\text { Meta-analisi non effettuata. } \\
\text { Adesione al trattamento: } 9 \text { studi in favore di collaborative care. } \\
\text { Miglioramento dei sintomi o remissione: tutti gli studi a favore della } \\
\text { collaborative care. }\end{array}$ \\
\hline $\begin{array}{l}\text { Neumeyer-Gromen } \\
\text { et al. } 2004\end{array}$ & 10 USA & 4196 & $\begin{array}{l}\text { Assistenza collaborativa (disease } \\
\text { management programs - DMPs) vs } \\
\text { Assistenza }\end{array}$ & $\begin{array}{l}\text { Non miglioramento sintomi di depressione }(\mathrm{N}=10) \text { : RR } 0.75 \text { (IC } 95 \% \\
0.70-0.81 \text { ) } \\
\text { Mancata adesione al trattamento - follow up } 90 \text { giorni }(\mathrm{N}=8) \text { : RR: } 0.59 \\
\text { (IC } 95 \% 0.46-0.75 \text { ) }\end{array}$ \\
\hline Bower \& Gilbody, 2006 & $\begin{array}{l}34 \text { (27 USA, } 4 \text { UK, } 1 \text { Olanda, } \\
1 \text { Cile, } 1 \text { Svezia) }\end{array}$ & 12294 & $\begin{array}{l}\text { Assistenza collaborativa vs Assistenza } \\
\text { abituale }\end{array}$ & $\begin{array}{l}\text { Uso di antidepressivi }(\mathrm{N}=28) \text { : OR } 1.92 \text { (IC } 95 \% \text { 1.54-2.39) } \\
\text { Miglioramento sintomi ( } \mathrm{N}=34 \text { ): SMD } 0.24 \text { (IC 95\% 0.17-0.32) }\end{array}$ \\
\hline Gensichen et al. 2006 & 13 (la maggior parte in USA) & $\begin{array}{l}\text { Non } \\
\text { riportato }\end{array}$ & $\begin{array}{l}\text { Assistenza collaborativa (case } \\
\text { management) vs Assistenza abituale }\end{array}$ & $\begin{array}{l}\text { Gravità sintomi dopo 6-12 mesi (N=11): SMD: }-0.40 \text { (IC } 95 \%-0.60-- \\
\text { 0.20) } \\
\text { Remissione dopo 6-12 mesi }(\mathrm{N}=7) \text { : RR: } 1.39 \text { (IC } 95 \% 1.30-1.48 \\
\text { Adesione al trattamento }(\mathrm{N}=9 \text { ): RR } 1.5 \text { (IC } 95 \% 1.28-1.86 \text { ) }\end{array}$ \\
\hline Gilbody et al. 2006 & 35 (28 USA, 7 Altro) & 12355 & $\begin{array}{l}\text { Assistenza collaborativa vs Assistenza } \\
\text { abituale }\end{array}$ & $\begin{array}{l}\text { Miglioramento outcome di depressione a } 6 \text { mesi }(\mathrm{N}=35) \text { : SMD } 0.25 \text { (IC } \\
95 \% 0.18-0.32 \text { ); } \\
\text { Miglioramento outcome di depressione a lungo termine: } \\
12 \text { mesi }(\mathrm{N}=6) \text { - SMD } 0.31 \text { (IC } 95 \% 0.01-0.53 \text { ); } \\
18 \text { mesi }(\mathrm{N}=5) \text { - SMD } 0.25 \text { (IC } 95 \% 0.03-0.46 \text { ); } \\
24 \text { mesi }(\mathrm{N}=9) \text { - SMD } 0.15 \text { (IC } 95 \%-0.03-0.34 \text { ); } \\
5 \text { anni }(\mathrm{N}=2) \text { - SMD } 0.15 \text { (IC } 95 \% 0.001-0.30 \text { ); }\end{array}$ \\
\hline Gunn et al. 2006 & 11 (10 USA, 1 UK) & & $\begin{array}{l}\text { Assistenza collaborativa vs Assistenza } \\
\text { abituale }\end{array}$ & $\begin{array}{l}\text { Meta-analisi non effettuata. } 8 / 11 \text { studi hanno evidenziato un aumento } \\
\text { statisticamente significativo della \% di pazienti migliorati o con } \\
\text { remissione a diversi peridi di follow up (range: } 10 \%-33 \% \text { ). }\end{array}$ \\
\hline Williams et al. 2007 & $\begin{array}{l}28 \text { (23 USA, } 1 \text { America } \\
\text { Latina, } 4 \text { Europa } \\
\text { Occidentale) }\end{array}$ & 10910 & $\begin{array}{l}\text { Assistenza collaborativa } \\
\text { (multicomponent intervention) vs } \\
\text { Assistenza abituale }\end{array}$ & $\begin{array}{l}\text { Meta-analisi non effettuata. } 16 / 23 \text { studi riportano un miglioramento dei } \\
\text { sintomi depressivi. } 15 / 23 \text { studi riportano un aumento del tasso } \\
\text { assoluto di antidepressivi prescritti: mediana } 17.8 \% \text { (range } 9.6 \%- \\
50.8 \% \text { ). }\end{array}$ \\
\hline Christensen et al. 2008 & 55 (38 USA, 12 UK, 5 Altro). & NA & $\begin{array}{l}\text { Assistenza collaborativa (case } \\
\text { management) vs Assistenza abituale }\end{array}$ & $\begin{array}{l}\text { Meta-analisi non effettuata. Componenti dell'assistenza integrata più } \\
\text { efficaci: monitoraggio sistematico del paziente con feedback regolari: } \\
\text { 8/11 studi, terapia cognitivo comportamentale effettuata da colui che } \\
\text { segue regolarmente il paziente:5/5 studi, preferenze del paziente } \\
\text { incorporate nel programma di cura: } 5 / 5 \text { studi; altre componenti che }\end{array}$ \\
\hline
\end{tabular}


rischio di risposta al trattamento (OR 2.30, CI95\% 10.35.05). Gensichen et al. (2006) misura la gravità dei sintomi (SMD: -0.40 , CI95\% -0.60--0.20). Badamgarav et al. (2003), Bower et al. (2006), Gilbody et al. (2006) misurano il miglioramento dei sintomi (SMD: 0.33, CI95\% 0.16-0.49, 0.24, C195\% 0.17-0.32, 0.25, CI95\% $0.18-0.32)$.

Tutte le revisioni che non effettuano meta-analisi riportano risultati in favore della assistenza integrata sia sulla remissione che sul miglioramento dei sintomi: 12/12 studi in Vergouwen et al. (2003), 8/11 studi in Gunn et al. (2006), 16/23 studi in Williams et al (2007).

In Christensen et al. (2008) le componenti dell'intervento che risultano maggiormente efficaci sono: monitoraggio sistematico del paziente con feedback regolari: 8/11 studi, terapia cognitivo comportamentale effettuata da colui che segue regolarmente il paziente:5/5 studi, preferenze del paziente incorporate nel programma di cura: $5 / 5$ studi; altre componenti che risultano efficaci, seppur in una percentuale minore di studi, sono: formazione del paziente e training per l'auto aiuto:14/28 studi, implementazione di linee guida: 9/20 studi, altri programmi di formazione per gli operatori sanitari: 9/17 studi (Tab 3).

\section{Discussione}

Tutte le revisioni individuate riportano risultati statisticamente significativi a favore dell'assistenza integrata rispetto all'assistenza abituale offerta dal solo medico di medicina generale sui differenti esiti considerati: riduzione o miglioramento dei sintomi, frequenza di remissione, adesione al trattamento. L'assistenza integrata risulta essere più efficace sia a breve che a lungo termine. Soltanto quattro revisioni (Badamgarav et al., 2003; Neumeyer-Gromen et al., 2004; Williams et al., 2007; Chang-Quan et al., 2009) valutano anche i costi della assistenza collaborativa.

Tutte evidenziano che i costi sanitari sono superiori rispetto alla assistenza usuale (utilizzo più elevato di farmaci e servizi), anche se in alcuni studi le differenze sono minime e non statisticamente significative.

I vantaggi economici in altre aree (giorni "depressionfree", con recupero sociale e lavorativo) sono stati calcolati in un solo RCT e devono, quindi, essere maggiormente approfonditi.

Di fronte a risultati così coerenti e così convincenti non resta che sviluppare le migliori strategie organizzative che permettano la massima trasferibilità nella pratica assistenziale reale di questi modelli di assistenza integrata. Affinché ciò accada, in tempi ragionevolmente brevi, coloro che coordinano l'assistenza sanitaria territoriale devono tenere presenti alcuni elementi che possono essere di facilitazione, o di 


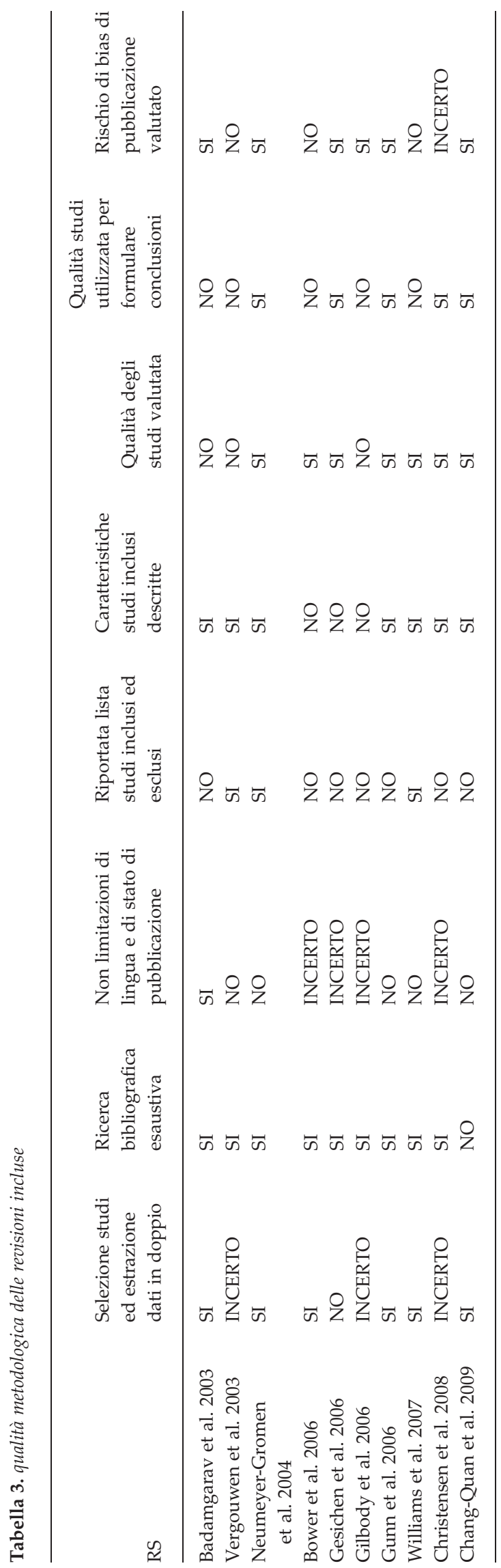

ostacolo, alla implementazione di tali strategie. Ne citiamo solo alcuni:

1. la transizione dai modelli centrati su un medico di medicina generale che lavora in solitudine a quelli di una vera e propria medicina di gruppo (che dispone di altre figure sanitarie esperte e di un coordinamento non episodico con i servizi specialistici territoriali) sta avvenendo molto gradualmente e ha lasciato spazio ad opzioni intermedie (ad es. integrazione degli orari di presenza, senza effettiva condivisione delle strategie) che non sembrano idonee a fare propri i modelli di "collaborative care" qui illustrati;

2. i servizi di salute mentale territoriali, a loro volta, non sembrano aver ancora messo a punto (se non episodicamente) una opzione organizzativa interna che si adatti ai tempi rapidi di un trattamento integrato dei disturbi depressivi. Quello che la "collaborative care" prefigura è infatti, a nostro avviso, un modello di "equipe territoriale trasversale" che abbia la capacità di coordinarsi rapidamente (entro un paio di settimane) e per un breve periodo di tempo (4-6 mesi). Il trattamento integrato è già pratica comune nei Centri di Salute Mentale, ma - nel caso dei disturbi psicotici - gli operatori della salute mentale lo propongono muovendosi come dei mezzofondisti o dei fondisti (molti mesi e/o anni); una "collaborative care" per i disturbi depressivi richiede invece prestazioni da velocisti (poche settimane e/o mesi), che implica modalità di allenamento e di attivazione ancora poco diffusi;

3. le strategie che puntano ad un miglioramento delle capacità di riconoscimento precoce dei singoli medici di medicina generale hanno dimostrato di non avere conseguenze positive sul miglioramento degli esiti della depressione.(Gilbody et al., 2008) Le capacità di riconoscimento devono - e possono essere migliorate, ma tali strategie formative producono risultati favorevoli solo se entrano a far parte di strategie di "collaborative care" (Christensen et al., 2008) . Lo stesso discorso vale per gli interventi mirati al monitoraggio ed al miglioramento dei livelli di adesione al trattamento (Bollini et al., 2006). Senza il coinvolgimento diretto, e coordinato, di altre figure sanitarie formate alle funzioni di riconoscimento e di monitoraggio non sembra remunerativo un ulteriore investimento formativo destinato ai soli medici di medicina generale.

L'efficacia della "collaborative care" sembra infine una conferma, sul piano organizzativo, e nei contesti della medicina generale, di quanto è già stato dimostrato come più efficace sul piano del trattamento individuale dei disturbi depressivi: il trattamento integrato (Pampallona et al., 2004). Un trattamento, cioè, che 
affianca - sempre - al farmaco altri interventi non farmacologici (formativi, psicologici, di supporto all'adesione). Le strategie di trattamento "monodimensionali" della depressione, sia nella medicina generale che nei servizi specialistici (di psichiatria e di neurologia) non vanno ulteriormente sostenute, né a livello formativo né nelle occasioni di aggiornamento professionale.

\section{Ringraziamenti}

Gli autori ringraziano la Sig.ra Giusy Petruzzelli, bibliotecaria presso l'Istituto Mario Negri di Milano, per l'aiuto offerto per il reperimento del materiale bibliografico

\author{
S. Minozzi ${ }^{1}$, G. Tibaldi ${ }^{2}$, E. Parmelli ${ }^{3}$, V. Pistotti ${ }^{1}$, \\ L. Ferrannini ${ }^{4}$ and C. Munizza ${ }^{2}$ \\ ${ }^{1}$ Centro Cochrane Italiano, Istituto Mario Negri, Milano \\ ${ }^{2}$ Centro Studi e Ricerche in Psichiatria, ASLTO2, \\ Corso Svizzera, 165, 10149, Torino, Italia. \\ Fax: +390114395843 / +39011852936 \\ e-mail: cmunizza@tin.it \\ ${ }^{3}$ Università degli Studi di Modena e Reggio Emilia, Modena \\ ${ }^{4}$ Dipartimento Salute Mentale e Dipendenze, ASL3 \\ "Genovese", Genova
}

\section{Bibliografia}

Adli M, Bauer M \& Rush AJ (2006). Algorithms and collaborative-care systems for depression: are they effective and why? A systematic review. Biol Psychiatry 59, 1029-1038

Badamgarav E, Weingarten SR, Henning JM, Knight K, Hasselblad V, Gano A Jr., \& Ofman JJ (2003). Effectiveness of disease management programs in depression: a systematic review. Am J Psychiatry 160, 2080-2090

Barbui C \& Tansella M (2006). Identification and management of depression in primary care settings. A meta-review of evidence. Epidemiologia e Psichiatria Sociale 15, 276-283

Bollini P, Pampallona S, Kupelnick B, Tibaldi G \& Munizza C (2006). Improving compliance in depression: a systematic review of narrative reviews. J Clin Pharm Ther 31, 253-60

Bower P, Gilbody S, Richards D, Fletcher J \& Sutton A (2006). Collaborative care for depression in primary care. Making sense of a complex intervention: systematic review and meta-regression. $\mathrm{Br} J$ Psychiatry 189, 484-493

Chang-Quan H, Bi-Rong D, Zhen-Chan L, Yuan Z Yu-Sheng P \& Qing-Xiu L (2009). Collaborative care interventions for depression in the elderly: a systematic review of randomized controlled trials. J Investig Med 57, 446-55

Christensen H, Griffiths KM, Gulliver A, Clack D Kljakovic M \& Wells L (2008). Models in the delivery of depression care: a systematic review of randomised and controlled intervention trials. BMC Fam Pract 9, 25

Craven MA \& Bland R (2006). Better practices in collaborative mental health care: an analysis of the evidence base. Can J Psychiatry 51, 7S-72S

Fletcher J, Bower PJ, Gilbody S, Lovell K, Richards D \& Gask L (2007). Collaborative care for depression and anxiety problems in primary care (Protocol). Cochrane Database of Systematic Reviews, Issue 2. Art. No.: CD006525. DOI: 10.1002/14651858.CD006525

Gensichen J, Beyer M, Muth C, Gerlach FM, Von Korff M \& Ormel J (2006). Case management to improve major depression in primary health care: a systematic review. Psychol Med 36, 7-14

Gilbody S, Sheldon T \& House A (2008). Screening and case-finding instruments for depression: a meta-analysis. CMAJ 178, 997-1003

Gilbody S, Whitty P, Grimshaw J \& Thomas R (2003). Educational and organizational interventions to improve the management of depression in primary care: a systematic review. JAMA 289, 3145-3151

Gilbody S, Bower P, Fletcher J, Richards D \& Sutton AJ (2006). Collaborative care for depression: a cumulative meta-analysis and review of longer-term outcomes. Arch Intern Med 166, 2314-21

Gunn J, Diggens J, Hegarty K \& Blashki G (2006). A systematic review of complex system interventions designed to increase recovery from depression in primary care. BMC Health Serv Res 6, 88

Katon W\&.Unutzer J (2006). Collaborative care models for depression: Time to move from evidence to practice. Archives of Internal Medicine 166, 2304-2306

Lopez AD, Mathers CD, Ezzati M, Jamison DT \& Murray CJ (2006). Global and regional burden of disease and risk factors, 2001: systematic analysis of population health data. Lancet 367, 1747-1757

NICE - National Institute for Clinical Excellence (2004). Depression: the Management of Depression in Primary and Secondary Care. National Institute for Clinical Excellence: London

NHS Centre for Reviews and Dissemination (2002). Improving the recognition and management of depression in primary care. Effective Health Care Bulletin 7, 1-11

Neumeyer-Gromen A, Lampert T, Stark K \& Kallischnigg G (2004). Disease management programs for depression: a systematic review and meta-analysis of randomized controlled trials. Med Care 42, 1211-21

O'Connor D, Green S \& Higgins J (2008). Defining the review question and developing criteria for including studies. In Cochrane handbook for Systematic Reviews of Interventions (ed JPT Higgins and S Green), pp. 83-94. Wiley-Blackwell: Chichester, UK

Pampallona S, Bollini P, Tibaldi G, Kupelnick B \& Munizza C (2004). Combined pharmacotherapy and psychological treatment for depression: a systematic review. Arch Gen Psychiatry 61, 714-719

Sartorius N (2001). The economic and social burden of depression. J Clin Psychiatry 62 Suppl 15, 8-11 
Shea BJ, Grimshaw J, M.Wells GA, Boers M, Andersson N, Hamel C, Porter AC, Tugwell P, Moher D \& Bouter LM (2007). Development of AMSTAR: a measurement tool to assess the methodological quality of systematic reviews. BMC Med Res Methodol 7: 10

Singleton N, Bumpstead R, O'Brien M, Lee A \& Meltzer HY (2000). Office of National Statistics: Psychiatric Morbidity Among Adults Living in Private Households. Her Majesty's Stationery Office: London
Vergouwen AC, Bakker A, Katon WJ, Verheij TJ \& Koerselman F (2003). Improving adherence to antidepressants: a systematic review of interventions. J Clin Psychiatry 64: 1415-1420

Williams JW Jr., Gerrity M, Holsinger T, Dobscha S, Gaynes B \& Dietrich A (2007). Systematic review of multifaceted interventions to improve depression care. Gen Hosp Psychiatry 29: 91-116 